\title{
Intelligent Control Method and Self-position-azimuth Correction Method for Autonomous Vehicle
}

\author{
Takuya Nishimuraa ${ }^{a}$ Sho Asai ${ }^{b}$, Liuyang Song ${ }^{c}$ and Peng Chen ${ }^{\mathrm{d}^{*}}$ \\ Mie University, Mie 514-8507, Japan \\ a515m222@m.mie-u.ac.jp, b514M204@m.mie-u.ac.jp, ‘512D202@bio.mie-u.ac.jp \\ d*chen@bio.mie-u.ac.jp
}

Keywords: Autonomous vehicle, Fuzzy-neural network, Self-position identification

\begin{abstract}
This paper proposes an intelligent control system using fuzzy-neural network (FNN) for autonomous vehicle. The autonomous vehicle will appropriately and automatically recognize and judge the running environment and run along a given orbit by using FNN. In order to acquire the training data of FNN, the driving knowledge is extracted from the human driving data. Then, the driving data is processed and normalized by rough set and defuzzy method. Moreover, a self-positionazimuth method is proposed to detect and correct the position and azimuth of the vehicle by outsideworld information from stereo camera and laser sensor. The effectivity of the methods has been verified by tests using a model vehicle.
\end{abstract}

\section{Introduction}

Recently, in the industrial plant, production facilities are becoming automatic and intelligence. Therefore, the demand for the robot which can inspect and examine the production facilities with autonomous running is increasing. Therefore, the intelligent control method for autonomous vehicle for the inspection robot running automatically is also needed in large plant[1][2].

The purpose of this research is to construct an intelligent control system by using fuzzy-neural network (FNN) for an autonomous vehicle which can appropriately and automatically recognize and judge the running environment and can run along a given orbit speedy and efficiently. Besides, the dead reckoning method is frequently used to estimate the position of the autonomous vehicle. But there are accumulated errors by the road-surface roughness, the difference of rotating speed and the slip of the wheel. To resolve this problem, a stereo camera and laser sensor are used intermittently as an outside-world information to recognize the error between the estimated position and the actual measured position of the vehicle to realize self-position-azimuth.

\section{Construction Method}

Model Vehicle. The model vehicle (called RoboCarTM) used in the experiment is shown in Fig. 1. The dimensions of the model vehicle are W195.0 mm/D429.0 mm/H212.2 mm. The speed control and steering angle control can be carried out by the rotary encoder and the servo motor respectively.

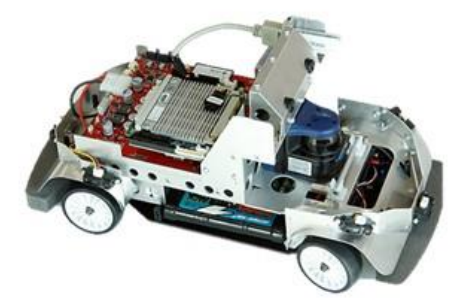

Fig. 1, RoboCar 
Vehicle Communication Environment. The model vehicle equipped with the Linux system and can be operated by other PC through access point. The model vehicle can automatically run after the program has been installed.

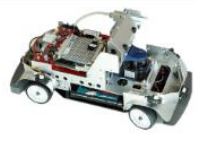

RoboCar

(Linux)

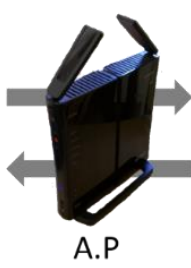

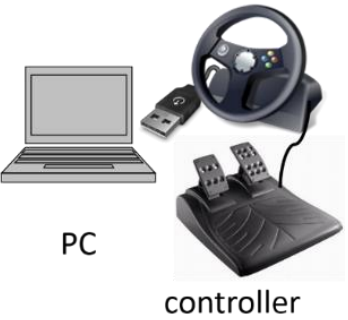

controller

Fig. 2, Construction of the model vehicle system

Setting Target Orbit. The target orbit is sampled by the points with the interval of several ten centimeters. The straight line between two points on the orbit is regarded as the approximate orbit. Then, these points for the orbit are memorized by the computer of the model vehicle. The relative distance between the vehicle and the points on the target orbit are sequentially calculated while autonomous running. The point with the smallest relative distance is seen as the previous target orbit point, the next point is regarded as the now target orbit point. And the following point is seen as the next target orbit point, as shown in Fig. 3.

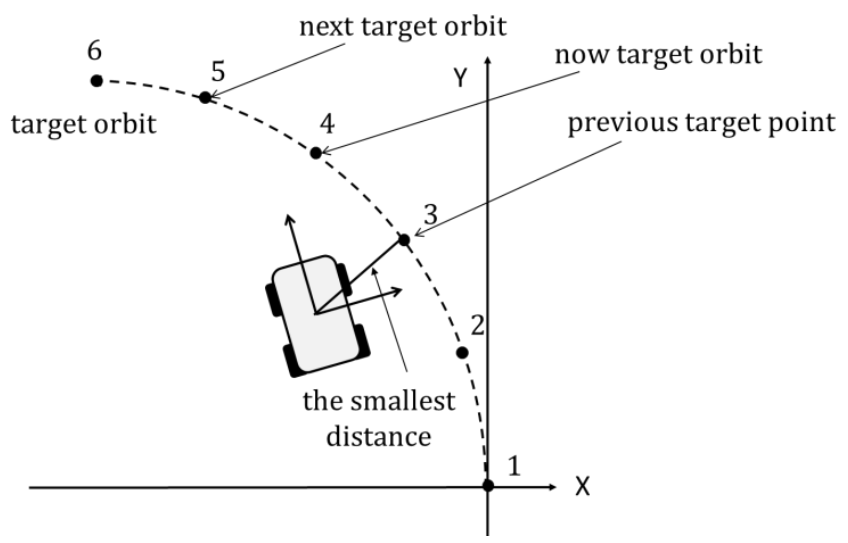

Fig. 3, Recognition of the orbit

Relative Position Parameters. Relative position parameters between the vehicle and target orbit as shown in Fig. 4. $P_{\mathrm{a}}$ is the relative distance between the center point of the vehicle and the target orbit. $P_{\mathrm{b}}$ and $P \mathrm{c}$ are the relative angles between the center line on the direction of the vehicle and the line connecting the cross points and the center point of the vehicle. Cross points are the intersections of the target orbit and the circle centered on the vehicle with different radius. Cross point 1 is the intersection of the target orbit and the circle whose radius is $30 \mathrm{~cm}$. Cross point 2 is the intersection of the target orbit and the circle whose radius is $60 \mathrm{~cm}$. The vehicle can recognize a relative position by using these relative position parameters. 


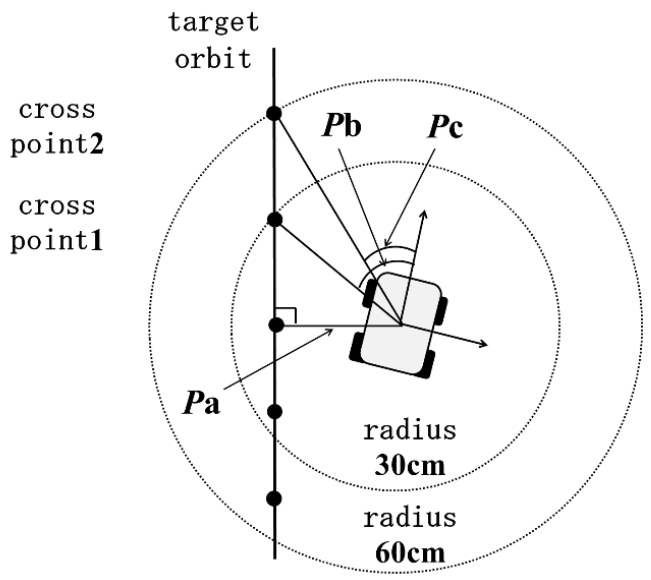

Fig. 4, Relative parameters

Method for extracting driving knowledge. Firstly, the running data is recorded while the vehicle is driven along target orbit by a human driver. $P_{\mathrm{a}}, P_{\mathrm{b}}, P_{\mathrm{c}}$ and steering angle are acquired as the running data. Then, the running data is processed and normalized by rough set and defuzzy method. Human's driving knowledge (teaching data for the FNN) is extracted from the running data.

Normalization. Firstly, we need to normalize $P_{\mathrm{a}}, P_{\mathrm{b}}, P_{\mathrm{c}}$ and steering angle for processing the teaching data by rough set. The range of the normalized relative distance and angle is narrow when the vehicle position is near to the target orbit, else it is wide when the vehicle position is distant from the target orbit. Steering angle is normalized with the interval of 3 degree.

Table 1, Range of normalization distance

\begin{tabular}{cccc}
\hline Parameters $\mathrm{Pa}[\mathrm{cm}]$ & $\mathrm{Pb}[\mathrm{deg}]$ & $\mathrm{Pc}[\mathrm{deg}]$ \\
\hline 1 & $\sim-30$ & $\sim-90$ & $\sim-90$ \\
2 & $-30 \sim-20$ & $-90 \sim-70$ & $-90 \sim-70$ \\
3 & $-20 \sim-10$ & $-70 \sim-55$ & $-70 \sim-55$ \\
4 & $-10 \sim-6$ & $-55 \sim-40$ & $-55 \sim-40$ \\
5 & $-6 \sim-3$ & $-40 \sim-30$ & $-40 \sim-30$ \\
6 & $-3 \sim-1$ & $-30 \sim-20$ & $-30 \sim-20$ \\
7 & $-1 \sim 1$ & $-20 \sim-15$ & $-20 \sim-15$ \\
8 & $1 \sim 3$ & $-15 \sim-10$ & $-15 \sim-10$ \\
9 & $3 \sim 6$ & $-10 \sim-6$ & $-10 \sim-6$ \\
10 & $6 \sim 10$ & $-6 \sim-3$ & $-6 \sim-3$ \\
11 & $10 \sim 20$ & $-3 \sim-1$ & $-3 \sim-1$ \\
12 & $20 \sim 30$ & $-1 \sim 1$ & $-1 \sim 1$ \\
13 & $30 \sim$ & $1 \sim 3$ & $1 \sim 3$ \\
14 & & $3 \sim 6$ & $3 \sim 6$ \\
15 & & $6 \sim 10$ & $6 \sim 10$ \\
16 & & $10 \sim 15$ & $10 \sim 15$ \\
17 & & $15 \sim 20$ & $15 \sim 20$ \\
18 & & $20 \sim 30$ & $20 \sim 30$ \\
19 & & $30 \sim 40$ & $30 \sim 40$ \\
20 & & $40 \sim 55$ & $40 \sim 55$ \\
21 & & $55 \sim 70$ & $55 \sim 70$ \\
22 & & $70 \sim 90$ & $70 \sim 90$ \\
23 & & $90 \sim$ & $90 \sim$ \\
\hline
\end{tabular}

Rough set. In this section, we explain the rough set used this research[3]. As a simple example, the data set is shown in Table 2. $P$ (the set of inputted parameters) is shown by Eq. $1 . S_{k}$ shown in Table 2 is outputted state after inputting the set $P$.

$$
P=\{a, b, c\}
$$

The $\mathrm{i}^{\text {th }}$ set of parameter value is shown by Eq. 2 . 


$$
\begin{aligned}
& { }^{1} P=\{3,2,1\} \\
& { }^{2} P=\{3,4,1\} \\
& { }^{\cdots} P=\{1,4,1\}
\end{aligned}
$$

\begin{tabular}{|c|c|c|c|c|}
\hline Data & \multicolumn{3}{|c|}{ Parameters P } & $S k$ \\
\hline No & a & $\mathrm{b}$ & g & \\
\hline $\mathrm{X}_{1}$ & 3 & 2 & 1 & 0 \\
\hline $\mathrm{X}_{2}$ & 3 & 4 & 1 & 1 \\
\hline $\mathrm{X}_{3}$ & 1 & $T$ & 3 & 0 \\
\hline $\mathrm{X}_{4}$ & 1 & 4 & 1 & 0 \\
\hline $\mathrm{X}_{5}$ & 3 & 4 & 1 & 1 \\
\hline $\mathrm{X}_{6}$ & 1 & 4 & 1 & 1 \\
\hline $\mathrm{X}_{7}$ & 4 & 6 & 2 & 0 \\
\hline $\mathrm{X}_{8}$ & 3 & 2 & 1 & 0 \\
\hline $\mathrm{X}_{9}$ & 4 & 6 & 2 & 1 \\
\hline $\mathrm{X}_{10}$ & 1 & 4 & 1 & 1 \\
\hline
\end{tabular}

Table 2, Parameters before processed by rough set

Approximation space set A is shown by Eq. 3 .

$$
A=\left\{r_{1}, r_{2}, r_{3}, r_{4}, r_{5}\right\}=\left\{\left\{X_{1}, X_{8},\right\},\left\{X_{2}, X_{5},\right\},\left\{X_{3}\right\},\left\{X_{4}, X_{6}, X_{10}\right\},\left\{X_{7}, X_{9}\right\}\right\}
$$

Then,

$$
\begin{aligned}
& r_{1}=\{3,2,1\},{ }^{\mathrm{r} 1} S=\{0,0\} \\
& r_{2}=\{3,4,1\},{ }^{\mathrm{r} 2} S=\{1,1\} \\
& r_{3}=\{1,7,3\},{ }^{\mathrm{r} 3} S=\{0\} \\
& r_{4}=\{1,4,1\},{ }^{\mathrm{r} 4} S=\{0,1,1\} \\
& r_{5}=\{4,6,2\},{ }^{\mathrm{r} 5} S=\{0,1\}
\end{aligned}
$$

By Eq. $5,{ }^{S k} \beta_{r i}$, uncertainty of measurement to state $S_{k}$ of equivalent class $r_{i}$, are

$$
\begin{aligned}
& { }^{0} \beta_{r 1}=2 / 2=1: 100 \% \\
& { }^{1} \beta_{r 1}=0 / 2=0: 0 \% \\
& { }^{0} \beta_{r 2}=0 / 2=0: 0 \% \\
& { }^{1} \beta_{r 2}=2 / 2=1: 100 \% \\
& { }^{0} \beta_{r 3}=1 / 1=1: 100 \% \\
& { }^{1} \beta_{r 3}=0 / 1=0: 0 \% \\
& { }^{0} \beta_{r 4}=1 / 3=0.33: 33 \% \\
& { }^{1} \beta_{r 4}=2 / 3=0.67: 67 \% \\
& { }^{0} \beta_{r 5}=1 / 2=0.5: 50 \% \\
& { }^{1} \beta_{r 5}=1 / 2=0.5: 50 \%
\end{aligned}
$$

Table 3 shows the result processed by rough set which can be leant by the FNN. 
Table 3, Result processed by rough set

\begin{tabular}{|c|c|c|c|c|c|}
\hline \multirow[t]{2}{*}{ rough sets $r_{i}$} & \multicolumn{2}{|c|}{ Probability } & \multicolumn{3}{|c|}{ Parameters $P$} \\
\hline & ${ }^{0} \beta_{\mathrm{ri}}$ & ${ }^{1} \beta_{\mathrm{ri}}$ & a & $b$ & $c$ \\
\hline 00 & & & & & \\
\hline $\mathrm{r}_{1}=\left\{\mathrm{X}_{1}, \mathrm{X}_{8}\right\}$ & $100 \%$ & $0 \%$ & 3 & 2 & 1 \\
\hline 11 & & & & & \\
\hline $\mathrm{r}_{2}=\left\{\mathrm{X}_{2}, \mathrm{X}_{5}\right\}$ & $0 \%$ & $100 \%$ & 3 & 4 & 1 \\
\hline 0 & & & & & \\
\hline $\mathrm{r}_{3}=\left\{\mathrm{X}_{3}\right\}$ & $100 \%$ & $0 \%$ & 1 & 7 & 3 \\
\hline $\begin{array}{lll}0 & 1 & 1\end{array}$ & & & & & \\
\hline $\mathrm{r}_{4}=\left\{\mathrm{X}_{4}, \mathrm{X}_{6}, \mathrm{X}_{10}\right\}$ & $33 \%$ & $67 \%$ & 1 & 4 & 1 \\
\hline $\begin{array}{ll}0 & 1\end{array}$ & & & & & \\
\hline$r_{5}=\left\{X_{7}, X_{9}\right\}$ & $50 \%$ & $50 \%$ & 4 & 6 & 2 \\
\hline
\end{tabular}

Fuzzy-Neural Network (FNN). The FNN which has leant the driving knowledge can control the vehicle to automatically and appropriately recognize and judge the running environment as human driver[3][4][5]. The FNN can output the appropriate results even if the input data have not been learnt beforehand. In this research, the inputs of the FNN are $P \mathrm{a}, P \mathrm{~b}$ and $P \mathrm{c}$, the output of the FNN is steering angle. The construction of the FNN is shown in Fig. 5.

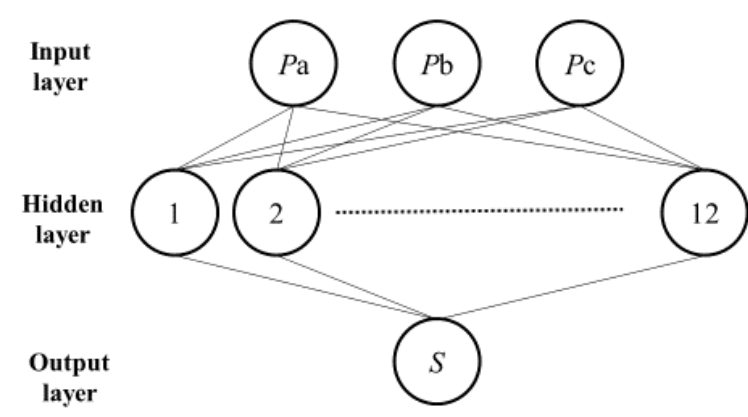

Fig. 5, Construction of FNN for orbit tracking

Acquisition the teaching data. In order to verify the effectivity of the methods, we set the target orbit which are consist of two straight routes and two half circles routes. The FNN are trained by the human-driving data acquired from 3 target orbit whose radius of the half circles routes are $57.5 \mathrm{~cm}$, $150 \mathrm{~cm}$ and $200 \mathrm{~cm}$. The initial positions are shown in Fig. 6.
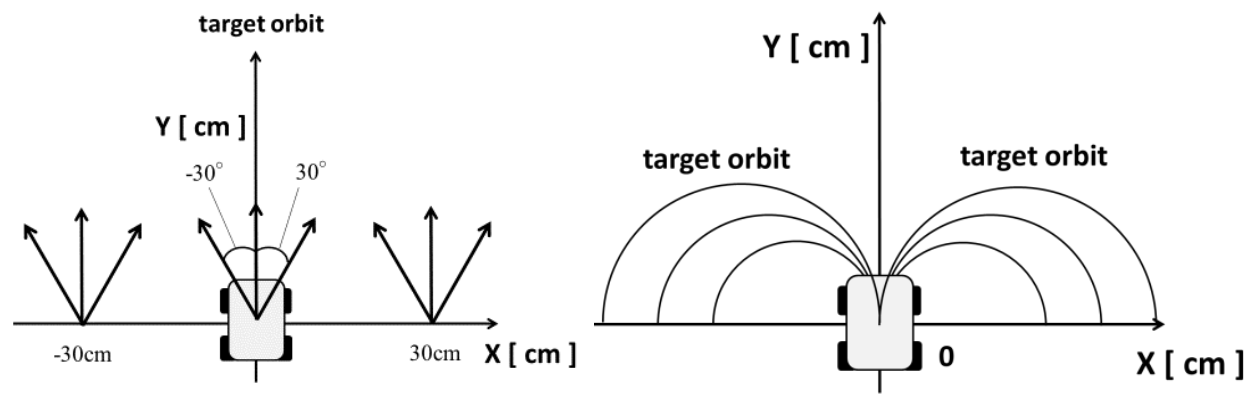

Fig. 6, The initial position of the vehicle 
The model vehicle's speed is $20 \mathrm{~cm} / \mathrm{s}$. The initial direction is 0 degree when running along the target orbits whose radius is $57.5 \mathrm{~cm}$ and $200 \mathrm{~cm}$. And when running along the target orbit whose radius is $150 \mathrm{~cm}$, the initial directions are set as 0,30 and -30 degrees.

Self-position-azimuth Correction Method. The autonomous vehicle generally recognizes selfposition using the dead-reckoning method. But there are accumelated errors by road-surface roughness, the slip of wheel and the error of sensers. The image information from stereo camera and angle information from laser sensor are used to resolve this probrem as self-position-azimuth correction. When the autonomous vehicle judges that there is a wall on the left side of the forward direction by the binary image data, the vihicle can acquire the distance data between the wall and the autonomous vehicle, and the angle data between the target orbit and the forward direction by using laser sensor. With these data, the autonomouse vehicle can perform self-position identification.

Image processing with the stereocamera. The autonomous vehicle can continuously acquire the binary image data shown in Fig. 7 for the self-position-azimuth correction while running, and in this case, the wall was recagnized for this purpose. The image is processed to recognize the black line on the wall at the left side. About the portion surrounded by broken line in Fig. 7, the white part is changed to 0 and the black part is changed to 1.The coordinates $x$ and $y$ are built using the binary data. Three quadrilateral arears with $\Delta x_{i}$ and $\Delta y_{i}$ shown in Fig. 8, and following equations are defined to recognize that the quadrilateral arears are if in the line on the wall.

$$
\begin{array}{r}
\sum_{x_{i}=X_{\mathrm{I}}, X_{\mathrm{II}}, X_{\mathrm{III}}}^{X_{\mathrm{I}}+\Delta x, X_{\mathrm{II}}+\Delta x, X_{\mathrm{III}}+\Delta x} \sum_{y_{i}=Y_{J}}^{Y_{J}+\Delta y} p\left(x_{i}, y_{i}\right)<P \\
x_{i}=1,2, \ldots, X_{N} ; y_{i}=1,2, \ldots, Y_{N}
\end{array}
$$

The symbols in the above equation are shown in Fig. 8, and $p\left(x_{i}, y_{i}\right)=0$ or 1 . If $\mathrm{P}$ is smaller than a threshold value, the quadrilateral arears are judged in the line on the wall. For example, in this case, $\Delta x_{i}=\Delta y_{i}=7, P=4$, and the center of the Three quadrilateral arears are less than the range of 5 along y-axis, as shown in Fig. 8, the image data at this portion is recognized as the image from a wall.

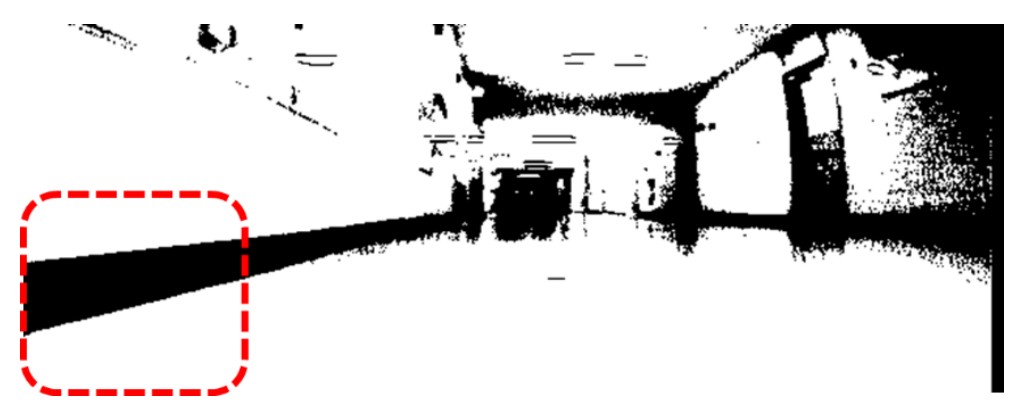

Fig. 7, Binalized image

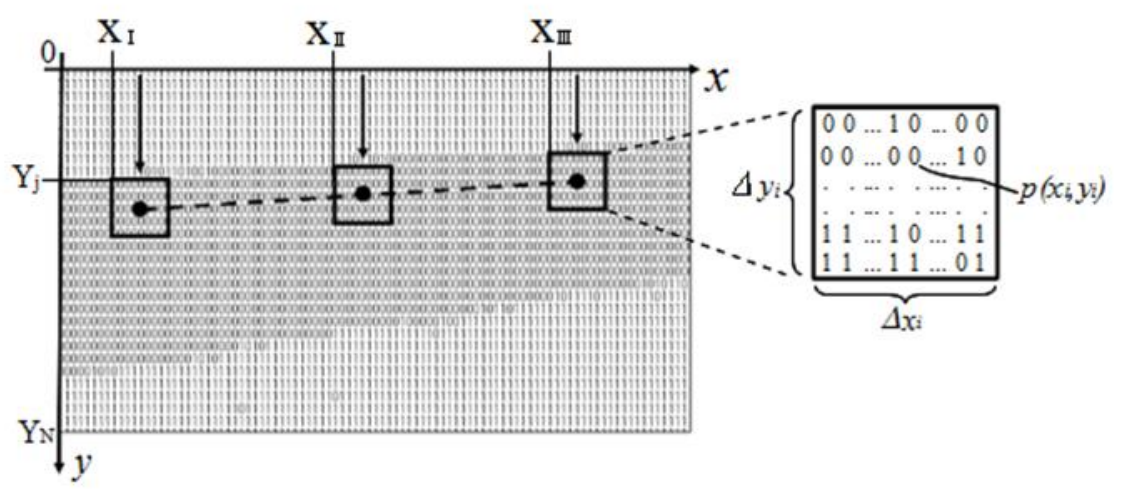

Fig. 8, Recognition of the line on the wall 
Self-position-azimuth Correction with laser sensor. By using laser sensor, the autonomouse vehicle can measure the distance between the wall and the vehicle, and the angle between the forward direction and the target orbit, and the measured data are input into the FNN at the same time. In this way the autonomouse vehicle identifies the error bettween the estimated position and the actual measured position. The distancesthat laser sensor can detect in 240 degree area are divided into 240 parts (dist $[i], i=1,1,2, \ldots, 240)$ with the interval 1 degree as shown in Fig. 9. The dist [i] shows the smallest distance to the wall. The direction angle $\theta$ of the vehicle can be calculated by Eq. 7 .

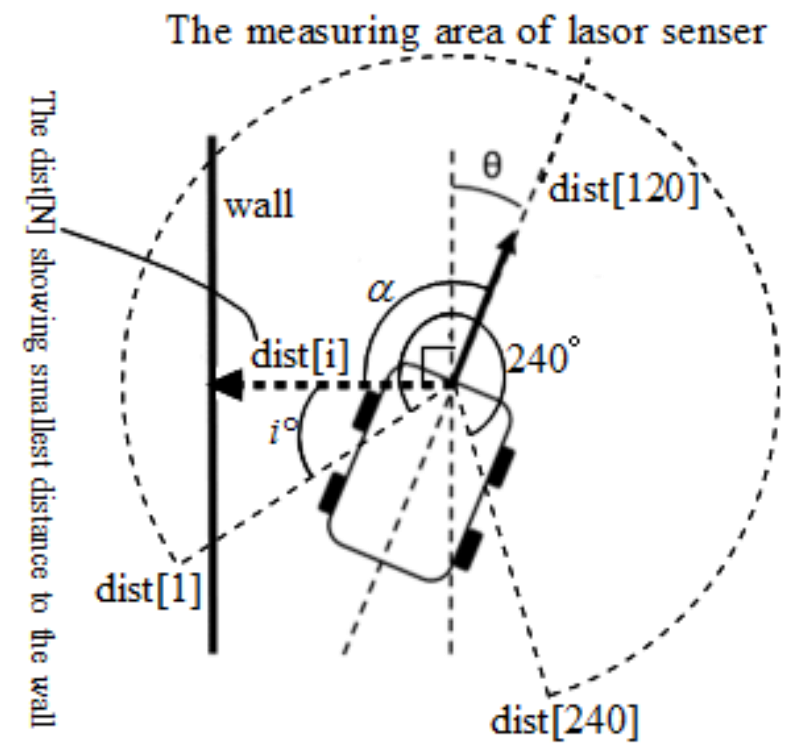

Fig. 9, Self-position-azimuth correction

$\alpha=120^{\circ}-i^{\circ}, \quad \theta=\alpha-90^{\circ}$

\section{Verification}

Experiment for autonomouse orbit tracking. The experiment for aoutmatically orbit tracking was carryied out through a buildingcorridors in the Mie University. The length of the orbit is about $240 \mathrm{~m}$ and consists of straight section and curve section. The autonomous vehicle runs along the target orbit keeping the distance about $0.85 \mathrm{~m}$ from the wall with the speed of $20 \mathrm{~cm} / \mathrm{s}$, and the initial angle was setting as 0 degree.

Result of autonomouse orbit traking. The result of experiment is shown in Fig. 10. The vehicle can run aoutomatically along the target orbit and recognizing self positon and azimuth using the image information from stereo camera and the angle information from laser sensor.

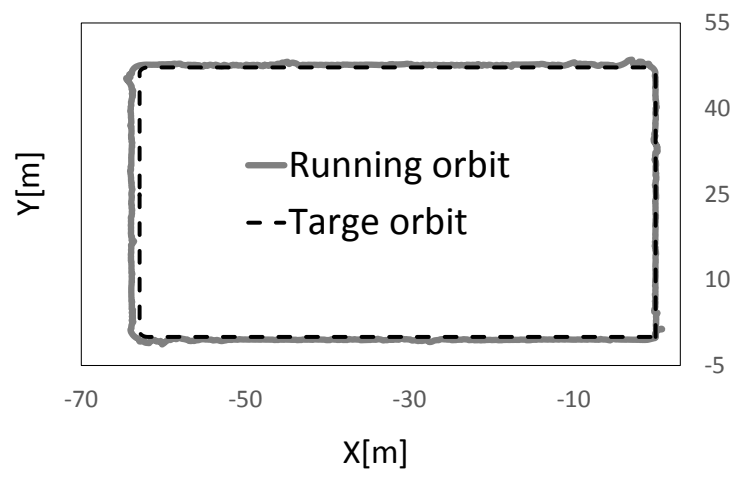

Fig. 10, The result of autonomous running experiment 


\section{Conclusion}

This paper proposed an intelligent control system using fuzzy-neural network (FNN) for autonomous vehicle. The autonomous vehicle can appropriately and automatically recognize and judge the running environment and can run along a given orbit speedy and efficiently by using FNN. In order to acquire the training data of FNN, the driving knowledge was extracted from the human driving data. The driving data was processed and normalized by rough set and defuzzy method. Moreover, a self-position-azimuth method was proposed to detect and correct the position and azimuth of the vehicle by outside-world information from stereo camera and laser sensor. The vertification experiments in this study show tha the vehicle can run long gavin orbit stably by selfposition-azimuth correction with image information from stereo camera, laser sensor and FNN. We will continue to reseach on the autonoumouse vehicle for automatically inspecting and diagnosing plant machinery.

\section{Reference}

[1] Peng Chen, Toshio Toyota and Yutaka Sasaki : Fuzzy diagnosis and fuzzy navigation for plant inspection and diagnosis robot, Proceedings of 1995 IEEE Int (Volume:1 ), pp.185-192, 1995.

[2] Peng Chen, Yutaka Sasaki and Toshio Toyota: Plant inspection and diagnosis robot for the detection of a faulty machine part by GA control, Intelligent Robots and Systems, 1998. Proceedings., (Volume:3, IEEE ), pp. 1664-1669, 1998.

[3] R. Takauji, P. Chen, and M. Yamashita, Intelligent Control Method for Autonomous Vehicle by Fuzzy-Neural Network and Self-position-azimuth Correction, AROB 17th, Japan, 2012.

[4] K. Yana, Y. Suzuki, Neuro information process technology, kaibundousyuppan, Japan, 1992, pp. 73-85.

[5] A. Okishio, Study on the fuzzy navigation of a mobile robot for outdoor works, Japan, 1995. 